\title{
La financiación de recursos en las MIPYME del sector comercial de Manizales (Colombia)
}

\author{
Funding for MSMEs resources in the commercial sector of Manizales \\ O financiamento dos recursos nas Mipyme do setor comercial de \\ Manizales (Colômbia)
}

Recibido el 15 de julio de 2015. Aceptado el 12 de abril de 2016

\author{
Gabriel Eduardo Escobar Arias* \\ Colombia \\ Rubén Darío Arango Álvarez** \\ Colombia
}

- Para citar este artículo:

Escobar Arias, Gabriel Eduardo

y Arango Álvarez, Rubén Darío

(junio, 2016). La financiación

de recursos en las MIPYME del

sector comercial de Manizales

(Colombia). Ánfora, 23(40), 149-

165. Universidad Autónoma de

Manizales. ISSN 0121-6538.

\section{Resumen}

Objetivo: determinar un sistema de amortización que se adecue a los recursos que disponen las Mipyme del sector comercial de Manizales. Metodología: se aplicó encuesta a 301 Mipyme y a 15 entidades del sector financiero de Manizales (Colombia). El proceso investigativo contó con tres fases: identificar las 301 empresas de forma aleatoria para la aplicación de la encuesta compuesta por las diferentes variables que inciden a la hora de demandar u ofertar los recursos monetarios, tasas de interés, los tiempos de colocación y los montos para créditos y beneficios económicos de Mipyme; luego, se encuestó el grupo de colocadores, es decir, a todas las entidades financieras que otorgan recursos. Finalmente, se indagó por la estructuración de

\footnotetext{
* Magíster en Administración Económica y Financiera, Economista, Profesor Universidad Autónoma de Manizales, Profesor Universidad Nacional de Colombia sede Manizales. Correo electrónico: gabrieledo@ autonoma.edu.co

** Magíster en Tributación, Contador público. Especialista en Finanzas. Profesor Universidad Autónoma de Manizales. Correo electrónico: rdarango@autonoma.edu.co
} 
las tasas de interés para casos específicos y cómo se identifican o miden los riesgos que generan este tipo de empresas. Resultados: respecto a las Mipymes se encontró que existen dificultades en el acceso a financiación de los recursos y cuando los obtienen tienden a una inadecuada utilización de estos, ya que se destinan a actividades. Se obtuvo un sistema de amortización escalonado donde los tomadores crediticios puedan tener una mayor flexibilidad en los flujos de caja de acuerdo con el periódico pago de sus cuotas. Conclusiones: en aquellos casos donde hay una relación importante entre prestador y empresario, el cobro por el crédito tiende a reducirse en la medida en que esta relación sea más larga, incluso, en estos casos, menos exigencias o garantías se requieren para el respaldo de los créditos.

Palabras Clave: Financiación, Empresas medianas y pequeñas, Recursos, Sistemas de amortización.

\section{Abstract}

Objective: to determine a suitable system of amortization for MSMEs available resources of the commercial sector of Manizales. Methodology: a survey was applied to 301 MSMEs at random and to 15 financial organizations of Manizales (Colombia). The research process included three phases, namely, identification of enterprises, implementation of the survey with different variables regarding the demand or the offer of monetary resources, interest rates, installation times and amounts for credit and economic benefits of MSMEs. A later survey was applied to the group of underwriters, that is, all financial institutions that grant resources. Finally, it was queried about the structure of interest rates for particular cases and how risks are identified and measured in such enterprises. Results: regarding MSMEs, results showed that there are difficulties when obtaining financing resources and if granted, they are used improperly. A tiered system of amortization where credit taker may have more flexibility in cash flows according to periodic payments. Conclusions: in cases where there is an important relationship between the provider and the entrepreneur, charging for credit tends to be reduced to the extent that this relationship is longer, even in these cases, fewer request or guarantees are required to backup those credits.

Keywords: Financing, Medium and Small Enterprises, Resources, Systems of amortization. 


\section{Resumo}

Objetivo: determinar um sistema de amortização que se adéque aos recursos que dispõem as Mipyme do setor comercial de Manizales. Metodologia: aplicouse pesquisa de opinião a 301 Mipyme dum jeito aleatório e a 15 entidades do setor financeiro de Manizales (Colômbia). O processo investigativo contou com três fases: identificação das empresas, aplicação da pesquisa composta pelas diferentes variáveis que incidem à hora de demandar ou ofertar os recursos monetários, taxas de interesse, os tempos de colocação e as quantidades para créditos e benefícios econômicos de Mipyme; logo, inquiriu-se o grupo de colocadores, quer dizer, a todas as entidades financeiras que outorgam recursos. Finalmente, indagou-se pela estruturação das taxas de interesse para casos específicos e como se identificam ou medem os riscos que envolvem este tipo de empresas. Resultados: respeito às Mipymes identificouse que existem dificuldades no acesso ao financiamento dos recursos e quando os obtêm tendem a uma inadequada utilização destes, já que se destinam às atividades. Obteve-se um sistema de amortização dividido onde os tomadores creditícios possam ter uma maior flexibilidade nos fluxos de caixa de acordo com o período de pago de suas parcelas. Conclusões: nos casos onde tem uma relação importante entre credor e empresário, a cobrança pelo crédito tende a se-reduzir na medida em que esta relação seja mais longa, inclusive, nestes casos, menos exigências ou garantias requerem-se para o respaldo dos créditos.

Palavras Chave: Financiamento, Empresas medianas e pequenas, Recursos, Sistemas de amortização. 


\section{Introducción}

Las MIPYME ${ }^{1}$ en Manizales son tema de discusión en los entornos económicos, productivos y sociales del país, ya que son base fuerte del crecimiento económico. Sin embargo, se han encontrado con el problema de la financiación para incrementar su motor productivo; esta dificultad se presenta en cualquier tipo de préstamo sin importar los plazos (Rodríguez, 2003).

La banca en Colombia está bajo el direccionamiento de negocio rentable que quiere obtener sus frutos. La alta rentabilidad que busca, hace que orienten sus créditos, principalmente, a vivienda, automóviles, construcción y libre consumo. Pero los créditos de inversión, necesarios para las MIPYMES, son menos ofertados ya que representan un riesgo más alto y menos rentabilidad para los bancos. De ahí, que los créditos sean otorgados más fácil para estos productos y no tanto para los de libre inversión, que requieren las MIPYMES; estos son créditos que tienen un riesgo bastante alto frente al respaldo de un negocio pequeño o mediano en crecimiento.

El sistema financiero es el motor económico para incrementar el desarrollo en todos los aspectos de un país (Aceves y Martínez, 2013). Colombia no es la excepción; en tal sistema, las entidades prestan dinero a personas de todas las clases sociales, pero a su vez son captadores número uno de grandes capitales de pequeñas o grandes empresas. Sin dejar a un lado los particulares, el negocio fundamental se encuentra en colocar ese dinero captado en inversiones rentables que generen dividendos en determinado tiempo. Existen en el mercado, entonces, la tasa de captación y la tasa de colocación; surge, así, una diferencia llamada tasa de intermediación financiera que genera ganancias a las entidades financieras. Es decir, el sistema se mueve bajo un círculo en el que se generan ganancias entre inversionistas, ahorradores y la banca.

Y al constituirse un negocio rentable, es necesaria una buena colocación de los capitales; esto se calcula a través del riesgo de cada uno de los agentes que necesita el dinero. El banco se asegura que no tendrá dificultades con la recuperación de los montos y los intereses.

\footnotetext{
1. Se entiende por micro, pequeña y mediana empresa, toda unidad de explotación económica, realizada por persona natural o jurídica, en actividades empresariales, agropecuarias, industriales, comerciales o de servicios, rural o urbana, que responda a los siguientes parámetros: Microempresa, a) Planta de personal no superior a 10 trabajadores b) Activos totales excluida la vivienda por valor inferior a 500 SMMLV. Pequeña empresa, a) Planta de personal entre 11 y 50 trabajadores. b) Activos totales por valor entre 501 y menos de 5.000 SMMLV. Mediana empresa, a) Planta de personal entre 51 y 200 trabajadores. b) Activos totales por valor entre 5.001 a 30.000 SMMLV.
} 
De acuerdo con lo anterior, aunque existe gran variedad de entidades en el sector financiero, no todas contemplan entre sus clientes a las MIPYMES, porque el riesgo es más alto. Los bancos, en su portafolio, ofrecen como primera opción el financiamiento de sus operaciones e inversiones aunque, muchas veces, el proceso para acceder a los capitales es extenso y no muy claro. En la mayoría de los casos, las tasas de interés varían para las MIPYME, además del alto riesgo, porque éstas no tienen un conocimiento profundo de la información financiera que deben aportar; es decir, las grandes empresas, al entregar información más clara, acceden a tasas de interés mucho más favorables que aquellas empresas que apenas están en proceso de crecimiento físico y productivo.

El documento de la CEPAL "Política pública e instrumentos de financiamiento a las MIPYME en Colombia” (Zuleta, 2011), resalta cómo

(...) las políticas de acceso al financiamiento en Colombia han buscado lograr mayor eficiencia en la asignación de los recursos públicos involucrados. Con ese objetivo, el enfoque de la política ha cambiado desde la asignación de crédito subsidiado, en las décadas de los ochenta y noventa, hacia el desarrollo de instrumentos que garanticen el acceso en condiciones de eficiencia, incorporando también al sector privado (p. 22).

La cita evidencia que en las dos últimas décadas la asignación de crédito a las MIPYME se ha venido realizando sin tasas subsidiadas, pero haciendo énfasis en los plazos y en las garantías.

La obtención de un crédito para el crecimiento y desarrollo de una empresa, en el caso de Colombia depende, en un porcentaje muy alto, de su historial crediticio. Pero además, en su capacidad de pago, reflejada en sus activos y su capital; por eso, en la mayoría de los casos las grandes empresas son las que tienen acceso directo a los capitales con grandes beneficios representados en las tasas de interés.

En cuanto a la bolsa de valores como opción para conseguir recursos a través de la emisión de acciones o venta de bonos, queda la duda. Sin embargo, hay que tener en cuenta que en ella sólo participan empresas con grandes capitales. Si en determinado caso las MIPYME pudieran hacer parte del portafolio que ofrece la bolsa de valores, serían pequeñas empresas que se dejarían a un lado por su riesgo; es decir, en cualquier ámbito las MIPYME generan más posibilidad de pérdida o una ganancia menor referente a las grandes empresas. 
En una entidad como la bolsa de valores se captan grandes capitales que se incrementan en el largo plazo, a través de la compra de acciones, principalmente. Pero, así como ocurre en el sector financiero, aquí el riesgo juega un papel más importante. Por lo tanto, los compradores dejan sus recursos en las acciones de las empresas más transadas y a la vez más rentables. Aproximadamente 100 grandes empresas cotizan en la bolsa de valores para obtener recursos de largo plazo, mediante la emisión de acciones o a través de bonos corporativos. De éstas, solamente 30 tranzan diariamente títulos; es decir, la diferencia de ellas no representa movimiento de oferta y demanda, debido a la gran concentración de la inversión en activos que representen menor riesgo para los inversionistas.

Emery y Finnerty (2000), demuestran cómo la bolsa de valores es una de las mejores maneras para obtener financiación en el largo plazo, ya que estos recursos se desembolsan con tiempos hasta de 30 años para su pago a diferencia de los bancos que en su mayoría otorgan los créditos con vencimiento hasta de 7 años. De acuerdo con lo anterior surge la pregunta, ¿cómo podría ser competitivo un pequeño empresario frente a uno grande si existen diferencias de acceso a los mecanismos de financiación?

En el 2011, a través de una encuesta realizada por ANIF (Asociación Nacional de Instituciones Financieras) en la cual se encuestaron 2.979 empresarios MIPYME, se determinó un descenso en las solicitudes de crédito de un 47\% en 2012 a un $36 \%$ en el segundo semestre de 2013 (ANIF, 2014), y aunque sí se dieron créditos se observó que estos fueron líneas de redescuento en su mayoría ofrecidas por Bancoldex ${ }^{2}$.

ANIF sostiene que las pequeñas y medianas empresas del país continúan experimentando dificultades para acceder al crédito bancario tradicional y por eso deben recurrir a fuentes alternativas de fondeo. De hecho, una encuesta realizada por el centro de estudios ANIF (2014) señala que el 49\% de las MIPYME en industria, comercio y servicios utiliza mecanismos alternativos para financiarse.

Algunos autores demuestran el difícil acceso a la financiación de las MIPYME:

Berger et al. (19ᄀ94), señalan cómo los grandes empresarios obtienen recursos a través del mercado público de valores a grandes plazos, mientras que las pequeñas

2. Bancoldex (Banco de Desarrollo Empresarial Colombiano) diseña y ofrece nuevos instrumentos, financieros y no financieros, para impulsar la competitividad, la productividad, el crecimiento y el desarrollo de las micro, pequeñas, medianas y grandes empresas colombianas, ya sean exportadoras o del mercado nacional. Ofrece instrumentos para promover la inclusión financiera en la población de menores ingresos por medio del programa de Inversión Banca de las Oportunidades. 
firmas lo hacen a través de intermediarios financieros; lo anterior basado en las brechas existentes entre las simetrías de información que se dan entre las distintas clases de empresas.

Boot y Thakor (2009), demostraron cómo a través del tiempo, de una mejora de la relación y conocimiento del cliente, las tasas de interés comenzaban a reducirse para las pequeñas firmas, sobre todo, cuando éstas presentaban proyectos en la banca.

Petersen y Rajan (1993) indican que la duración de la relación entre el banco y la empresa no induce alguna relación con el valor final del crédito, es decir, la tasa de interés no presentaba correlación estadística con la duración de la relación que se da entre la empresa y el agente crediticio.

Hellman y Lindsey (2003), muestran cómo los bancos en los Estados Unidos han sido partícipes en el capital de nuevos negocios, bajo la figura de capitalistas de riesgo; lo hacen con el objetivo de otorgar créditos a empresas de mediano o largo plazo en las que tengan participación en una mesa directiva. Así podrán ser partícipes en las decisiones y apoyar el crecimiento de estos nuevos negocios.

Myers y Majluf (1984), exponen cómo las empresas, para financiar sus proyectos, acuden al mercado público de valores; sin embargo, cuando hay información privilegiada en los mercados, los riesgos de emisión de acciones se aumentan ante una potencial caída de los precios de las mismas. Por tanto, algunos empresarios prefieren hacerlo con recursos propios en la medida que hay riesgos de información privilegiada que puede terminar en una disminución del valor de la empresa. Se señala también cómo las MIPYME no tienen acceso al mercado público para financiar sus inversiones, lo que ocasiona que tengan que recurrir a fuentes de financiación más costosas que hacen que el valor presente neto de sus inversiones se caiga o que demuestre la inviabilidad de los mismos.

Benavete et al. (2005), reconocen cómo las MIPYME no tienen buen acceso al crédito de las entidades financieras, ya que ellas prefieren disminuir los riesgos otorgándoles recursos a las grandes firmas; esto, basado no en el riesgo y en los plazos, sino más bien en la dinámica propia de la economía en la cual las firmas se deben estar renovando, permanentemente, por efectos del mercado cambiante en el cual se encuentran las organizaciones.

La teoría del Racionamiento del Crédito de Stiglitz y Weiss (1981), muestra que la mecánica del sistema financiero para el otorgamiento de los créditos está 
en función de la tasa de interés que esperan recibir y el riesgo que perciben a la hora de colocar los recursos. Estas tasas se pueden ver afectadas por dos aspectos: a) el efecto de selección adversa y b) las acciones de los prestatarios. Donde el efecto de la selección adversa opera de la siguiente manera: en la medida en que las empresas tienen diferentes mecanismos y formas para realizar el reembolso de sus obligaciones a las entidades financieras, éstas no pueden conocer fácilmente quiénes o qué empresas serán las buenas o malas para el pago de las deudas; por tanto, tendrá que utilizar diferentes mecanismos y estrategias para identificar el mejor cliente y es a este a quien le colocará los recursos.

De acuerdo con lo señalado por los autores anteriormente nombrados, se evidencia las dificultades que tienen este tipo de empresarios para acceder a recursos de las entidades financieras; entre éstas se encuentran la falta de información, los grandes riesgos, de los mercados en que operan estas empresas; por eso, en el proceso investigativo realizado se evidenció estos tipos de problemáticas, lo que generó la siguiente pregunta de investigación: ¿Qué sistema de amortización se adecúa a las necesidades de recursos de las MIPYME del sector comercial de Manizales?

Los objetivos en los cuales se centró la investigación fueron: inicialmente determinar un sistema de amortización de crédito adecuado para las MIPYME del sector comercial de Manizales, para ello se indagaron las necesidades de financiación que tienen las empresas de este sector; luego se realizó una descripción de los diferentes productos de financiación que le ofrecen las entidades colocadoras de recursos a las MIPYME y finalmente proponer una forma adecuada de financiación.

Este trabajo se refiere al estudio sobre el acceso a la financiación de recursos para las MIPYME como un aporte tanto al sector financiero colombiano y a las micro, pequeñas, medianas y grandes empresas que requieren comenzar procesos de modernización o de transformación institucional para acceder al mercado financiero. Esto, les apoya en la tarea de fondear, adecuadamente, sus proyectos de expansión, dado que los documentos solicitados por las entidades financieras, son muchos.

Esta investigación reflejó los aspectos que influyen en el desarrollo de las MIPYME del sector comercial de Manizales y ofrece alternativas a las necesidades de financiación de recursos que requieren. Así mismo, se muestra cómo el sector financiero presenta una serie de productos como capital de trabajo, libre inversión, tasas de interés, participación como propietario, entre otros, para que los 
empresarios dispongan de herramientas que faciliten la generación de estrategias de mercado de las MIPYME. Incluso, los resultados de esta investigación podrán ser utilizados por la banca comercial y por los empresarios, permitiéndoles focalizar en los aspectos que deben ser tenidos en cuenta al momento de presentar alternativas de financiación.

Con esta investigación se espera contribuir en parte a esa responsabilidad social empresarial que deben tener las entidades colocadoras de recursos, particularmente con las MIPYME que hacen parte del desarrollo de la economía colombiana.

\section{Metodología}

El estudio fue de corte descriptivo, ya que se elaboró un instrumento de encuesta tanto para las MIPYME como para el sector financiero. Fue tabulado a través del sistema Excel, arrojando respuestas que fueron debidamente agrupadas de acuerdo con la intención de la investigación. Tales resultados permitieron obtener información sobre las variables a tener en cuenta en la relación crediticia: plazos, tasas de interés, montos solicitados, la finalidad de los recursos, requerimientos, entre otras.

En Manizales se cuenta con una población de 1390 empresas del sector comercial; esta información fue proporcionada por la empresa Confa ${ }^{3}$ y obtenida mediante un convenio suscrito entre la Universidad Autónoma de Manizales y la entidad citada. Por lo tanto, la muestra de Mipyme MIPYME a la que se le aplicó la encuesta fue a 301, las cuales fueron el resultado estadístico de la siguiente formula:

$$
\begin{aligned}
& \mathrm{n}=[\mathrm{N} * \mathrm{Z} 2 *(\mathrm{P} * \mathrm{Q})] /[(\mathrm{N}-1) * \mathrm{E} 2+(\mathrm{P} * \mathrm{Q}) * \mathrm{Z} 2] \\
& \text { Donde: } \\
& \mathrm{N}=\text { Tamaño de la población } \\
& \mathrm{Z}=\text { Nivel de confianza (Para un nivel de confianza del } 95 \%) \\
& \mathrm{E}=\mathrm{E} \text { ) porcentaje de error aceptado } \\
& \mathrm{P}=\text { Probabilidad de éxito }
\end{aligned}
$$

\footnotetext{
3. CONFA (Caja de Compensación Familiar de Caldas). Hace parte del Sistema del Subsidio Familiar en Colombia, son personas jurídicas de derecho privado sin ánimo de lucro, organizadas como corporaciones. Cumplen una función social y se encuentran sometidas al control y vigilancia del Estado en la forma establecida por la Ley. Estas corporaciones funcionan gracias a los aportes que realizan las empresas afiliadas equivalentes al $4 \%$ sobre el valor total de su nómina; así desarrollan los diferentes programas y servicios.
} 
$\mathrm{Q}=$ Probabilidad de fracaso

$\mathrm{n}=$ Tamaño de la muestra a determinar

Desarrollando la fórmula arroja el siguiente resultado:

$\mathrm{n}=\left[1.390^{*}(1,96) 2^{*}(0,5 * 0,5)\right] /[(1.390-1) *(0,05) 2+(0,5 * 0,5) *(1,96) 2]$

$\mathrm{n}=1.334,4 / 4,4=301$

También se desarrolló otra encuesta de oferta financiera que fue aplicada a 15 entidades financieras de Manizales, la cual corresponde a la totalidad de la población.

Para tener una adecuada y sistemática recolección de información se realizó el proceso investigativo en tres fases o momentos: en primer lugar, se identificaron 301 empresas -de forma aleatoria para la aplicación de la encuesta- que se desenvuelven en el sector comercial de Manizales. Después se caracterizaron los agentes que intervienen en la problemática a estudiar. Se diseñaron dos encuestas con el objetivo de establecer cuales variables inciden a la hora de demandar u ofertar los recursos monetarios como las tasas de interés, los tiempos de colocación, los montos a solicitar.

Por eso, el grupo que se analizó fue el de los empresarios a quienes se les indagó sobre cómo obtienen los recursos; esto, principalmente para establecer una relación directa entre los montos y las tasas de interés que les son ofrecidas. Además, se preguntó sobre los principales obstáculos, desde la hora de definir montos, hasta los trámites para obtener los recursos.

Además se encuestó el grupo de colocadores; es decir, a todas las entidades financieras que otorgan recursos. Para este caso, se dio un enfoque en relación con el portafolio, para observar cuáles son los productos que son ofrecidos a las MIPYME. También se indagó sobre dos temas adicionales: uno de ellos, es la estructuración de las tasas de interés para casos específicos y, el otro, cómo identifican o miden los bancos el riesgo que puede generar este tipo de empresas.

\section{Resultados}

Se encontró que en cuanto a las empresas el mayor porcentaje es para las microempresas, con el $49.3 \%$. En segundo lugar, se encuentran las pequeñas empresas con el 41.4\%; las empresas consideradas grandes tan solo representan el $9 \%$. Es decir, que en un porcentaje muy alto el sector que estudia este trabajo, está compuesto por micro y pequeñas empresas, pues representan más del 90\% 
de todas las consideradas. También se encontró que los ingresos de las empresas del sector comercio oscila entre cero y mil millones de pesos, representando el $57.9 \%$ de las empresas encuestadas.

Sobre los tipos de obligaciones, el mayor número de créditos son solicitados para libre inversión alcanzando un 14\% del total de los encuestados; el 11\% de las empresas realizan solicitudes de créditos para hipoteca y compra de vehículo y en último lugar, las solicitudes son para el pago de tarjeta de crédito. Sobre las tasas de financiación se encontró que en promedio para estos créditos ascienden al $2 \%$ efectivo mes. También se evidenció que los créditos hipotecarios tienen plazos en un promedio de 10 años; para libre inversión y capital de trabajo es de 5 años.

Se indagó a las MIPYME sobre las garantías que les exige el sistema financiero para respaldar las deudas y se halló que el $33.7 \%$ respaldan sus obligaciones a través de activos como bienes inmuebles; en segundo lugar, se les exige un codeudor y, en tercer lugar, la pignoración de un bien mueble como lo manifiestan Berger y Udell (1994), quienes demostraron que la mayoría de entidades de crédito en sus operaciones de colocación exigen como garantía la pignoración de los activos fijos de las pequeñas firmas, con el objeto de disminuir los riesgos a la hora de prestar los recursos, Esta investigación se realizó con 3.400 pequeñas firmas.

En las encuestas realizadas a las entidades financieras se encontró que:

El 60\% de las entidades colocadoras de recursos indagadas, prestan dinero para capital de trabajo a las microempresas, aunque se determina que estas entidades prefieren las grandes empresas ${ }^{4}$.

El 33\% de las entidades colocadoras de recursos otorgan dinero para libre inversión a las microempresas y el 53\% para las grandes empresas.

Entre otros productos importantes se encuentran los créditos para tesorería. Se evidenció que solo el 20\% de las entidades financieras realizan estos préstamos a microempresas y el $73 \%$ a grandes empresas.

4. Se refiere a empresas donde el número de trabajadores se encuentra entre 101 hasta 251 y cuyos activos totales son superiores a 30.000 SMMLV. 
El 27\% de las entidades financieras prestan recursos para banca de fomento a las microempresas.

Sobre la pregunta del interés que tienen las entidades financieras de ser propietarias de aquellas empresas a las cuales les otorgan recursos, el $40 \%$ de ellas no están interesadas en este tipo de inversión; prefieren una garantía real crediticia frente a la posibilidad de ser partícipes en estas empresas.

El 100\% de las entidades indagadas, afirmó no estar interesadas en participar como accionistas en este tipo de empresas, para entregarles más fácil los créditos. Caso contrario con lo que ocurre en países como los Estados Unidos (Hellman et al, 2003) que demostraron que muchas entidades financieras están dispuestas a prestar los recursos a pequeñas firmas y son partícipes de las juntas directivas, con el objeto de asesorarlas para disminuir los riesgos del manejo de los recursos que tienen allí colocados.

El 13\% de las entidades financieras encuestadas están dispuestas a otorgar recursos a microempresarios a una tasa de interés del DTF más puntos adicionales; estos puntos adicionales son la prima por riesgo diferencial para cada microempresa.

El 53\% de las entidades que participaron en este estudio, tienen políticas establecidas de no otorgamiento de crédito a las microempresas por el riesgo que ello conlleva; el resto de entidades están dispuestas a otorgar recursos a este tipo de empresas, bajo la tasa de usura microcrédito la cual puede llegar $48 \%$ efectivo anual.

En cuanto a los tiempos que manejan para la recuperación de la inversión, es decir para el pago de los créditos, se aplica un promedio entre uno y cinco años de plazo para microempresas hasta grandes empresas. Así mismo, se otorgan excepciones como la línea de crédito para remodelación, que puede fluctuar entre uno y veinte años. Al otro extremo se encuentra el crédito de tesorería que tiene plazos reducidos que varían entre cero y un año

De las quince entidades indagadas, ocho no presentaron información sobre los plazos otorgados en los diferentes productos a las microempresas. Lo anterior demuestra que las tasas y los plazos se convierten en un obstáculo más para el crecimiento y desarrollo de las empresas. 
Según los datos y la consulta de autores plasmada en el marco teórico del presente trabajo, se evidencia que las empresas del sector comercial de Manizales presentan dificultades de acceso a la financiación de las entidades financieras por diferentes motivos. De acuerdo con lo anterior, se propone que los empresarios del sector, para acceder al crédito de una forma más fácil y tasas de interés más competitivas, deben presentar proyectos a las entidades financieras. Se espera que en la medida en que se dé un cumplimiento de las diferentes etapas de los proyectos, se comience a presentar una disminución escalonada en las tasas de interés del crédito asociadas al proyecto que se está ejecutando.

Para darle solución al problema planteado en este documento se realizó una comparación de diferentes sistemas de amortización de los créditos, utilizando en cada uno de ellos un mismo monto, una misma tasa de interés e igual plazo, encontrando que para cada uno de ellos se debe pagar la siguiente cantidad de intereses:

Tabla 1. Valor intereses a pagar para un crédito de $\$ 48.000 .000$ a una tasa de interés del 25,34\% E.A. a 5 años con pagos de cuotas mensuales para diferentes tipos de amortización.

\begin{tabular}{|c|c|}
\hline Sistema de amortización & V/r intereses a pagar \\
\hline Tasa fija sistema tradicional & $\$ 32.857 .764$ \\
\hline Tasa fija, cuotas crecientes & $\$ 34.305 .430$ \\
\hline Tasa fija, cuota creciente escalonada & $\$ 34.102 .249$ \\
\hline Cuota fija y tasa de interés escalonada & $\$ 32.493 .524$ \\
\hline
\end{tabular}

Fuente: elaboración propia

La tabla anterior resume los cálculos efectuados a un mismo crédito en las condiciones y plazos actuales; además, los resultados de esta investigación también evidencian que lo planteado (un sistema de amortización con cuota fija y tasa de interés escalonada) se convierte en una relación mucho más efectiva tanto para el sector financiero como para el empresario: en el juego ambos ganan y se incentiva al empresario a la presentación de nuevos proyectos de financiación. 
La propuesta se centra, en que los empresarios del sector puedan presentar un proyecto o plan de negocios, a la entidad financiera, que le generarían mayores rendimientos y utilidades a la empresa. Estos proyectos deben ser avalados por la entidad colocadora de los recursos y en la medida en que los empresarios logren las etapas planteadas en los mismos y estén cumpliendo con los pagos de las cuotas periódicas, la tasa de interés que se le aplica al crédito puede ir disminuyendo por los cumplimientos de las obligaciones del empresario. Con esto se demuestra el cumplimiento de lo establecido en la teoría como lo plantean Weston y Brighan (1981), con su teoría del Ciclo de Vida financiero; en ésta, en la medida en que se consolide un mejor conocimiento de la trayectoria de las empresas, las entidades colocadoras de los recursos pondrán a disposición y con menos exigencias mayores cantidades de recursos monetarios a las empresas para que puedan llevar a cabo sus proyectos.

Otros autores que respaldan esta forma de crédito como Boot y Thakor (2009) quienes señalan que los recursos se colocan más fácilmente y a menores tasas de interés en la medida en que haya un conocimiento mejor de los clientes. Por lo tanto, si las entidades financieras logran identificar estos buenos clientes y exista una mejor relación entre estos agentes, los recursos serán colocados con mayor facilidad y a menores costos financieros.

\section{Conclusiones}

El proceso investigativo arrojó información entre la relación existente en el momento de la solicitud, estudio y posible otorgamiento de recursos financieros a las MIPYME de los colocadores de créditos, desprendiéndose de ello las siguientes conclusiones:

En el sector empresarial de las MIPYME de Manizales son escasos los recursos financieros disponibles, por factores que dificultan el acceso a créditos financieros. Por lo tanto, al realizar encuestas a entidades financieras de la ciudad se encontró poca atracción del sector bancario para las MIPYME, dificultades de los empresarios para acceder a créditos financieros; de ahí el planteamiento de un sistema de amortización con cuota fija y tasa de interés escalonada, expuesto a continuación:

El sector bancario manizaleño no considera como nicho atractivo de mercado a las MIPYME, puesto que prefiere colocar sus recursos con alto grado de seguridad y bajo riesgo financiero en las medianas y grandes empresas. Esto se ve reflejado en el estudio llevado a cabo por (Benavete et al., 2005) donde reconocen cómo las MIPYME no tienen buen acceso al crédito de las entidades financieras, 
ya que ellas prefieren disminuir los riesgos otorgándoles recursos a las grandes firmas. Aunque Emery y Finnerty (2000), señalan cómo deben financiarse las empresas en el largo plazo a través del mercado público de valores. Para las micro, pequeñas y medianas empresas es casi imposible acceder a dicho mercado debido al alto riesgo que representan para los inversionistas, lo que hace que acceder a los recursos por esta otra vía no sea viable para esta tipo de empresas.

Benavete et al. (2005) afirman que las entidades financieras prefieren colocar sus recursos en empresas que tienen buena capacidad de pagar los préstamos con menor riesgo, aunque aquellas entidades que se atreven colocar recursos en MIPYME lo hacen a unas tasas de interés muy elevadas con el objeto de mitigar los riesgos que este tipo de empresas contiene. Para poder disminuir un poco los riesgos de colocación, las entidades financieras solicitan grandes respaldos para estas deudas como la garantía de un bien raíz, pignoración de un bien mueble, codeudor y la firma de pagaré.

En países más desarrollados, para disminuir los riesgos algunas entidades financieras lo que hacen es participar en las juntas directivas de empresas en las que colocan los recursos, con el objeto de administrar mejor el riesgo y asesorar los negocios para que puedan salir adelante, como lo muestran Hellmany Lindsey (2003). En Manizales, sin embargo, a los empresarios encuestados esta figura no les aplica, ya que los dueños de las MIPYME no están dispuestos a compartir la administración con terceros.

Otro problema que se evidenció en el proceso investigativo fue que los plazos de colocación de los recursos son a muy corto plazo para estas empresas, lo que dificulta aún más el crecimiento de las mismas. La colocación de los recursos, en promedio, oscilan entre 1 y 5 años; dicha condición no permite la relación con inversiones de largo plazo con retornos lentos, situación que hace más difícil el pago de las obligaciones, pues los flujos de caja no son suficientes en algunos casos para responder a las obligaciones.

Ante las dificultades encontradas por los empresarios para acceder a créditos se plantea un sistema de amortización de créditos en los cuales se genera un gana-gana tanto para otorgantes de los recursos como para los usuarios de los mismos. A través de un sistema de pagos con tasas escalonadas los colocadores de recursos seguirán recibiendo una buena cantidad de intereses que respaldan sus utilidades y mitigan los riesgos, como también los usuarios en la medida en que realicen sus pagos de forma adecuada se les otorgará una disminución de las tasa de interés, lo que aumenta su rentabilidad o mejora sus flujos de caja.

Para dar respuesta a la pregunta de investigación, el instrumento financiero que se propone dentro de la investigación es un sistema de amortización con cuota fija y tasa de interés escalonada, de forma tal que puedan ir disminuyendo en la medida en que las condiciones pactadas se cumplan entre las partes. Esto 
concuerda con el estudio desarrollado por Boot y Thakor (2009) en el que demostraron de una mejora de la relación y conocimiento del cliente, las tasas de interés comenzaban a reducirse para las pequeñas firmas. También demostraron que en la medida que las relaciones entre clientes y bancos fueran avanzando, las tasas de interés se reducían en la medida que continuaban según lo planeado.

Dicho sistema de amortización incentivará al deudor con disminución del pago total de los intereses a pagar. Por otro lado, esta modalidad permitirá que más recursos sean puestos a disposición de las micro, pequeñas y medianas empresas. Dicha propuesta también se encuentra en consonancia con Berger et al. (1994) cuando afirman que la relación entre los costos de la financiación está relacionada con la duración de la relación entre los agentes.

Finalmente, el estudio concluye que en aquellos casos donde hay una relación importante entre prestamista y empresario, el cobro por el crédito tiende a reducirse en la medida en que esta relación sea más larga, como también cuando la empresa disponga de mayor cantidad de activos, el riesgo percibido es menor y esto se refleja en la tasa de interés de los créditos. Se concluye, además, que en la medida que la relación banco-empresa sea más larga, menos exigencias o garantías se requieren para el respaldo de los créditos.

\section{Referencias}

Aceves, S. y Martínez, J. (2013). The financial system and his impact on the private sector. Dynamics, (58), 175-199.

ANIF. Encuesta a MIPYME, primer semestre de 2014. Recuperado el 16 de noviembre de 2014.

Benavete, J., Galetovic, A., y Sanhueza, R. (2005). La dinámica industrial y el financiamiento de las MIPYME. Trimestre económico, (72), 3-35.

Berger, N., Allen, U. y Gregory, F. (1994). Lines of credit and relationship lendingin small firm finance. Working Paper. New York: New York University.

Boot, A., y Thakor, A. (2009). The accelerating integration of banks and markets and its implications for regulation. Oxford, USA. Oxford University Press. 
Comisión Económica para América Latina y el Caribe (2014). Informe. Recuperado de www.cepal.org.

Emery, D., y Finnerty, J. (2000). Administración financiera corporativa. Ciudad de México: Pearson Educación.

Hellman F, T., y Lindsey L, A. (2003). Building Relationships Early: Banks in Venture Capital. Sauder School of Business Working Paper.

Hellman, T. (2002). A theory of estrategic venture investing. Journal of Financial Economics, 284-314.

Myers C, S. y Majluf S. N. (1984). Corporate financing and investment decisions when firms have information that investors do not have. Journal of Financial Economics, 13, 00 .

Petersen, M. y Rajan, R. (1993). The benefits of lending relationships: evidence from small business. 3-37.

Rodríguez, A., (2003). La realidad de la MIPYME Colombiana, desafío para el desarrollo. Bogotá, Colombia: Fundes Internacional.

Stiglitz E, J., y Weiss, A. (1981). Credit rationing in markets with imperfect information. The American Economic Review.

Superintendencia Financiera de Colombia (2014). Capitulo II Sistema de Administración de Riesgos.

Weston, E. y Brigham, J. (1981). Managerial finance. EE.UU: Dryden Press.

Zuleta, L. A. (2014). Política pública e instrumentos de financiamiento a las MIPYME en Colombia. 